\section{BIOORTHOGONAL CHEMISTRY}

\section{Click on, click off}

J. Am. Chem. Soc. doi:10.1021/jacs.7b05125 (2017)

Chemoselectivity is a key consideration when selecting from the toolbox of bioorthogonal chemical ('click') reactions for site-specific tagging of biological molecules, and new reactions are continually sought to enable precise, controllable labeling of multiple sites in tandem. Addy et al. have now added to this toolbox a chemoselective rapid azocoupling reaction (CRACR) that uses an aromatic diazonium ion to conjugate a desired tag to a particular aromatic amino acid residue. In the optimized reaction, the 4-carboxybenzenediazonium $(4 \mathrm{CDz})$ ion reacts rapidly with the readily available noncanonical amino acid 5-hydroxytryptophan (5HTP) and, importantly, exhibits selectivity over canonical aromatic amino acids such as tyrosine. The use of this noncanonical amino acid, which can be site-specifically incorporated into a desired protein via nonsense suppression technology, also endows the reaction with an added degree of selectivity by limiting the number of sites within the proteome where the reactive handle is present. An added benefit of this reaction is that it is 'unclickable' as well: addition of dithionite reductively cleaves the azo-coupled conjugate, thus providing a means to remove the tag. Although this CRACR reaction has not yet been shown to be compatible with other click reactions, its selectivity and reversibility make it a potentially attractive choice for future labeling applications.

\section{CHEMICAL METHODS}

\section{DREADDs see no CNO} Science 357, 503-507 (2017)

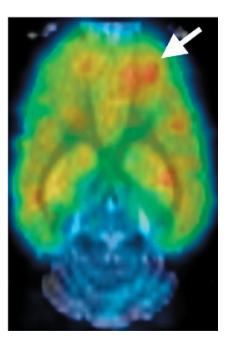

DREADD technology is widely used to manipulate neuronal activity in whole animals, and it involves 'designer' receptors based on human muscarinic receptors hM3Dq or hM4Di that have been mutated to be specifically activated by an inert and inactive metabolite of clozapine, $\mathrm{CNO}$, but not endogenous ligands. Unfortunately, $\mathrm{CNO}$ is converted to clozapine in vivo, thus complicating analysis and the clinical use of DREADDs. To assess the extent of this liability and to understand the mechanism of action of CNO at DREADD receptors, Gomez et al. have tested CNO binding and activity in cells and rodent brain tissue by using ligand-binding assays and autoradiography. Clozapine bind and activate DREADDs, but CNO do so only at concentrations much greater than those generally used in DREADD experiments. PET imaging has revealed substantial brain uptake of clozapine but not $\mathrm{CNO}$, thus suggesting that a combination of CNO conversion to clozapine, high clozapine brain permeability and highaffinity clozapine binding accounts for DREADD activation. Further experiments

DEVELOPMENTAL SIGNALING

\title{
Peaking early
}

Dev. Cell doi:10.1016/j.devcel.2017.07.014 (2017)

During Caenorhabditis elegans development, a graded EGFR-Ras-ERK signal is received by the six vulval precursor cells (VPCs). The P6.p cell receives the highest ERK signal, initiating formation of the vulva, whereas the neighboring VPCs, P5.p and P7.p, receive lower levels and adopt the secondary vulval fate. Fluorescent transcriptional reporters to detect ERK activity are limited, owing to the lag time between activation and fluorescence. de la Cova et al. have explored an alternative approach using kinase translocation reporters (KTRs), which translate the ERK phosphorylation status into the nucleocytoplasmic localization of a fluorescent reporter. The authors have modified an established ERK KTR for use in C. elegans by creating a biscistronic transgene containing the ERK KTR with an mCherry-H2B nuclear marker enabling detection of ERK activity by measuring the ratio of red/green signals in the nucleus. Analysis of fluorescence during vulval induction revealed multiple activity pulses in P6.p cells relative to the neighboring VPCs, in agreement with observations of frequency-modulated signaling. Altogether, this sensor system provides an exciting opportunity to detect ERK signaling with improved resolution in C. elegans and a potential to expand to other developmental organisms.

supporting this model have shown that the biological effects of DREADD activation require an $\sim 100$-fold-higher dose of $\mathrm{CNO}$ than clozapine, an effect aligned with the extent of conversion of CNO to clozapine. These results suggest that DREADDs require subthreshold clozapine levels for activation and may be useful as CNS disease therapeutics.

PROTEIN ENGINEERING

\section{Expanding splicing versatility} Proc. Natl. Acad. Sci. USA 114, 8538-8543 (2017)

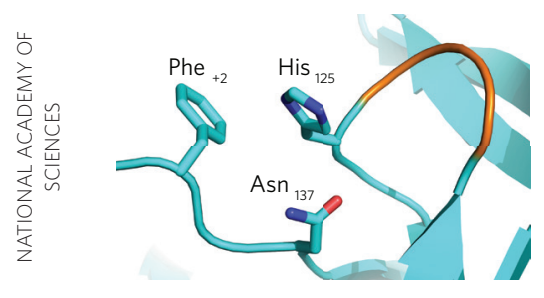

Cyanobacterial DnaE split inteins catalyze the linkage of two polypeptide chains in ultrafast protein trans-splicing reactions, an activity that is exploited in numerous protein engineering applications. However, sequence constraints near the splice site in the second polypeptide chain (C-extein) are still a limiting factor, because large hydrophobic residues at $\mathrm{C}$-extein position +2 are necessary to stabilize the side chain of the catalytically important histidine. Stevens et al., rationalizing that altering side chain dynamics might help to overcome these limitations, have mutated the three loop residues preceding the histidine in the Nostoc punctiforme DnaE split intein Npu and analyzed the mutants in an Escherichia coli selection assay. The $\mathrm{Npu}_{\mathrm{GEP}}$ loop mutant shows increased splicing rates for unfavorable C-extein +2 residues, and molecular dynamics simulations suggest that the conformational dynamics of the histidine side chain is indeed restricted in $\mathrm{Npu}_{\mathrm{GEP}}$. The authors have also introduced the GEP-loop mutation into their previously engineered stable consensus DnaE intein Cfa. The $\mathrm{Cfa}_{\mathrm{GEP}}$ enzyme displays improved protein cyclization activity and facilitates higher yields for in nucleo histone semisynthesis. This mechanism-guided mutagenesis approach may also be applied to other split inteins, thereby potentially expanding the applicability of these important and versatile protein engineering tools. 